\title{
Damage Assessment in a SiC-fiber reinforced Ceramic Matrix Composite
}

\author{
Konstantinos G. Dassios and Theodore E. Matikas \\ Department of Materials Science and Engineering, University of Ioannina, 45110 Ioannina, Greece \\ Correspondence should be addressed to Konstantinos G. Dassios; kdassios@cc.uoi.gr
}

Received 29 August 2012; Accepted 23 October 2012

Academic Editor: Sheng-Rui Jian

Copyright (c) 2013 K. G. Dassios and T. E. Matikas. This is an open access article distributed under the Creative Commons Attribution License, which permits unrestricted use, distribution, and reproduction in any medium, provided the original work is properly cited.

\begin{abstract}
Assessment of the fracture behavior of a SiC-fbre-reinforced barium osumilite (BMAS) ceramic matrix composite tested under static and cyclic tension conditions is reported herein. Notched specimens were used in order to limit material damage within a predefined gauge length. Imposition of successive unloading/reloading loops was found to result in an increase by $20 \%$ in material strength as compared to pure tension; the observed increase is attributed to energy dissipation from large-scale interfacial debonding phenomena that dominated the post-elastic tensile behaviour of the composite. Cyclic loading also helped establish the axial residual stress state of the fibres in the composite of tensile nature via a well-defined common intersection point of unloadingreloading cycles. A translation vector approach in the stress-strain plane was successful in establishing the residual stress-free properties of the composite and in reconciling the scatter noted in elastic properties of specimens with respect to theoretical expectations.
\end{abstract}

\section{Introduction}

Ceramic matrix composites (CMCs) with continuous reinforcements first appeared in the 1990s and offered optimized properties to the brittle-matrix materials such as increased fracture toughness, crack growth resistance, damage tolerance and strength, and decreased brittleness. These materials are less prone to unstable catastrophic failure than the first generations of CMCs mainly as a result of the damage mechanisms that develop during their fracture and consume part of the externally applied energy, hence decreasing the energy apportioned to the catastrophic work of crack advance at the crack tip. Today, continuously reinforced CMCs have effectively replaced ceramics and metals in applications with increased thermomechanical loading demands such as aircraft brakes, internal chambers and nozzles of jet motors, thermal barriers, turbine and burner nozzles, and space shuttle parts.

The mechanical behaviour of continuously reinforced CMCs depends on the properties of the matrix and fibers, on fibre aspect ratio and volume fraction, and, most importantly, on the properties of the interface, the fiber-matrix boundary that occupies a vast surface area of the material and is responsible for transferring the stresses from the continuous phase to the reinforcements. A mismatch between the coefficients of thermal expansion (CTE) of the matrix and the fibres is usual in composites of heterogeneous constituents. Then, upon cooling of the composite from the high manufacturing temperatures to ambient values, fibres can be found in residual tension if their CTE is higher than that of the matrix, or in residual compression, in the inverse scenario. Residual stresses also affect fiber/matrix interface conditions by inducing microcracks in the matrix material. Irrespective of the sign of residual state, the sheer existence of opposite stress levels between the composite constituents has a significant effect on the stress transfer characteristics at the interface. It is then expected that during testing of a composite with constituents of different CTEs, the extent of interfacial damage is directly associated with the thermal residual stresses (TRS) state of the material. Interfacial properties, mainly strength and hence efficiency, are also dependent on processing conditions. For example, it is 
known that during hot pressing of SiC-fibre-reinforced glassceramic matrix composites, a chemical reaction between the fiber surface and the oxides in the matrix results in the formation of a carbon-rich interfacial bond $[1,2]$, which is weak enough to result-especially in combination with a brittle matrix-in fiber-bridged matrix cracks when the composite is loaded in tension.

In cyclic loading tests, a common intersection point (CIP) may be formed by the compliance slopes of reloadingunloading loops. It has been shown [3-5] that the stress coordinate of this point is directly related to the residual stress of fibers in the composite. It should be noted that the CIP approach is currently the only plausible method for evaluating TRS from mechanical test data.

The current study deals with the assessment of interfacial damage and calculation of residual stresses in a continuous $\mathrm{SiC}$-fibre reinforced barium osumilite $\left(\mathrm{BaMg}_{2} \mathrm{Al}_{6} \mathrm{Si}_{9} \mathrm{O}_{30}\right.$, BMAS) glass-ceramic matrix composite loaded in cyclic tension. The residual stress of the fibers was directly measured by interrogation of the CIP that was assembled by raw experimental data alone, and the information was used to validate a prevalent theoretical TRS model. In the triple-regime tensile behaviour associated with interfacial debonding and final material fracture, the effects of negative inelastic strain accumulation and material damage with respect to the elastic behaviour of fibers are discussed in the text.

\section{Experimental}

2.1. Materials and Specimens. The cross-ply SiC/BMAS composite laminates, $3 \mathrm{~mm}$ thick, consisted of silicon carbide "Tyranno" fibers. The nominal elastic modulus and strength of the Tyranno fibres, as quoted by the manufacturer, are $190 \mathrm{GPa}$ and $3.3 \mathrm{GPa}$, respectively. The composite processing technique consisted of: (i) desizing the Tyranno fiber bundles in a furnace, (ii) wetting the desized fibers in a slurry of the precursor glass frit, (iii) winding the fibers on a mandrel and allowing to dry for $20 \mathrm{~min}$, (iv) cutting and manually arranging the fibers in layers for the preparation of prepreg sheets, (v) stacking the sheets in $(0,90)_{4 s}$ sequence, (vi) burning the binder off, and (vii) hot pressing in a graphite die at $\sim 1,200^{\circ} \mathrm{C}$ for $10 \mathrm{~min}$. Final crystallization was achieved by heat treatment at $1,300^{\circ} \mathrm{C}$. Fiber volume fraction in the final product was 0.55 (Figure 1).

A CNC vertical machining center equipped with a diamond wafering blade was used to cut the laminates into rectangular beams of dimensions $100 \times 12 \times 3 \mathrm{~mm}^{3}(\mathrm{l} \times$ $\mathrm{w} \times \mathrm{t}$ ). The orientation of fibers in the external plies was chosen to be parallel to the loading axis $\left(0^{\circ}\right)$. Notches were prepared using the same equipment. Notch-to-width ratios (notched ligament fractions) of 0.35 and 0.2 were used, while unnotched "dogbone" specimens were prepared for pure tensile loading. Sets of three specimens were prepared for each case. All tensile and cyclic tension tests were carried out in displacement control with a crosshead rate of $0.2 \mathrm{~mm} / \mathrm{min}$ on a servohydraulic testing frame equipped with a $100 \mathrm{kN}$ load cell and hydraulic clamping grips. A clip-on axial extensometer equipped with knife-edged legs, gauge length

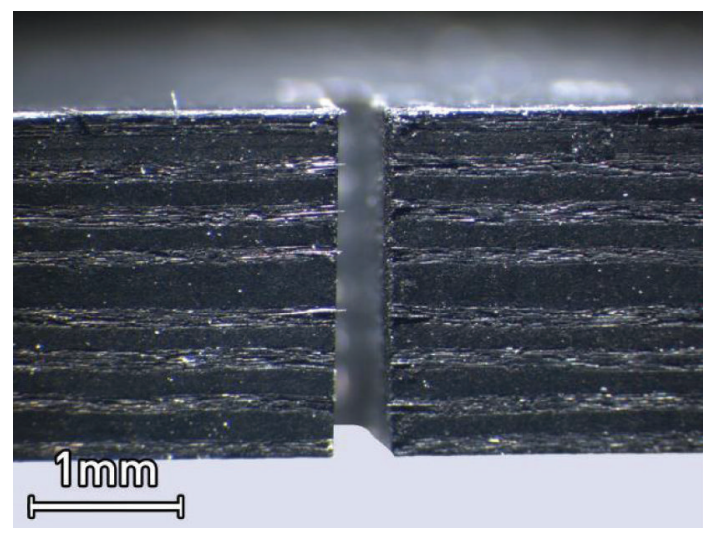

FIgURE 1: Stereoscopic side detail of a double-edge notched specimen.

$25 \mathrm{~mm}$, was used to externally capture strain. The initial strain rate, calculated for the effective gauge length of $50 \mathrm{~mm}$, was $4.0 \times 10^{-3} \mathrm{~min}^{-1}$. Unloading to complete relaxation and subsequent reloading cycles commenced at $0.1 \%$ strain with a step of $0.15 \%$ strain.

\section{Results and Discussion}

3.1. Mechanical Behaviour. Typical cyclic tension curves for three DEN specimens of different notch-to-width ratios of the SiC/BMAS composite are shown in Figure 2. Material response under monotonic tension is included in the bottom graph of Figure 2 as a dashed line. The stress-strain response of the composites consisted of an initial linear part, followed by a regime of gradually decreasing tangent modulus to the curve. The softening is due to interfacial debonding and slipping between matrix and fiber in the area confined between neighboring matrix blocks interconnected by fiber bundles, which are formed as a result of matrix cracking in planes perpendicular to the fibers' axis. The average slope of unloading-reloading cycles decreases with cycle count and suggests a degradation in material stiffness due to progressive matrix and interface damage. DEN specimens failed shortly after the attainment of the maximum load; hence, the associated curves exhibited very minimal tail effects.

Specimens without notches failed after a final regime of increased apparent stiffness and tangent modulus to the curve. Within this regime, large-scale debonding of the interface and matrix cracking have completed; the load bore by the matrix cannot increase further; hence, it is the fibers bear the global load. In this regime, the prevalent damage mechanism is load bearing by intact fibers, which is superior to those associated with the interface or the matrix; hence, a macroscopic stiffening becomes apparent, coupled with an almost linear stress-strain relationship. Catastrophic failure commences after failure of the first fiber bundle leading to a dramatic decrease in $V_{f}$. Similar stiffening phenomena and linear stress-strain end shapes in composite curves have also been reported by other researchers [6-8]. 


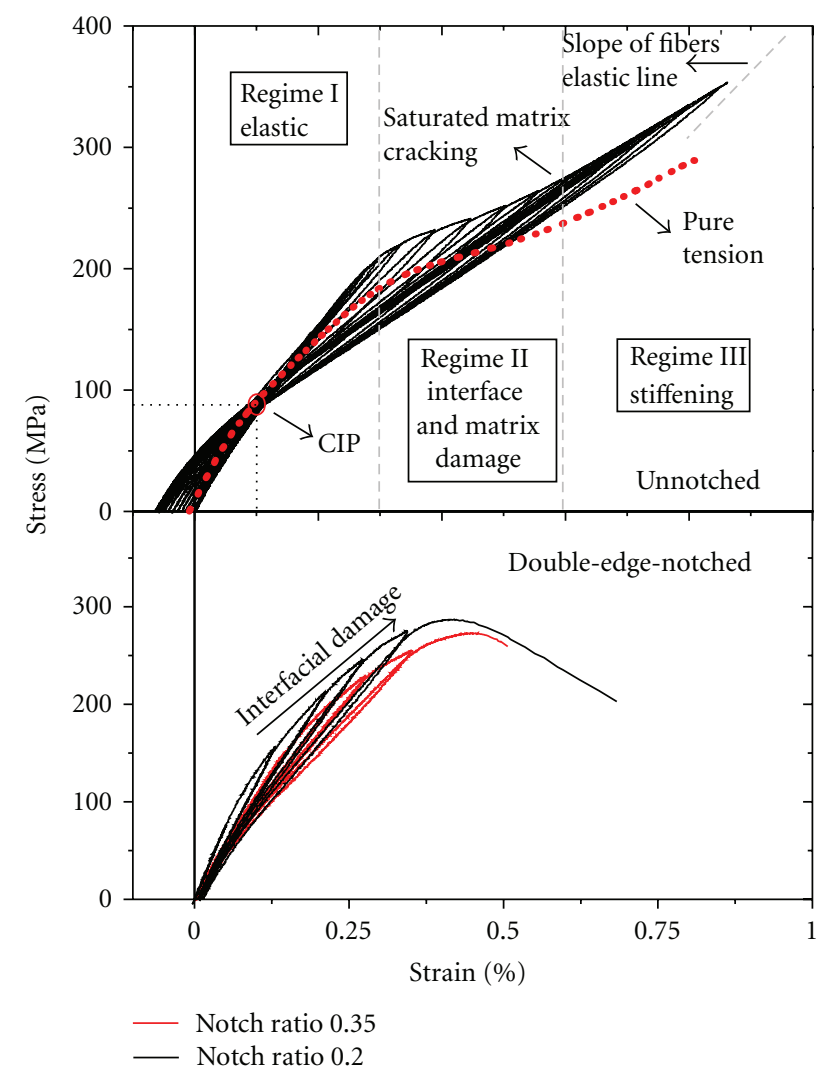

FIgURE 2: Typical mechanical response of the SiC/BMAS composite in tension for various initial notches.

In the case of unnotched specimens, the stress-strain curves exhibited a uniquely well-defined CIP of unloadingreloading curves in the first quadrant of the stress-strain curve (tensile domain) at $0.1 \%$ strain and $90 \mathrm{MPa}$ stress. In their studies, Steen [3], Camus et al. [4], and Mei [5], have established similar CIPs in the compression domain by extrapolation of the elastic compliance slopes of each reloading loop. By extrapolation, also Morscher [9] has calculated tensile residual stresses in $\mathrm{SiC}$ fibres in a $\mathrm{SiC}$ matrix. However, never before has a CIP been seen to experimentally assemble from raw experimental data alone. The tensile curves of notched specimens did not exhibit a CIP, as seen in Figure 2. Machining damage during notch fabrication may be one reason of cancelling of thermal residual stresses [10]. Another reason for TRS relaxation may be stress concentration starting at notch roots.

As far as mechanical properties are concerned, elastic modulus over the $25 \mathrm{~mm}$ gauge length appeared to decrease with increasing notch length; average values of 108, 119, and $151 \mathrm{GPa}$ were calculated for specimens with notch-to-width ratios of $0.35,0.2$, and 0 . The dependency of modulus on notch length is attributed to the finite dimensions of the gauge length compared to the stress concentration region. On the other hand, the composite modulus expected by the classical rule of mixtures using a matrix modulus of $E_{m}=120 \mathrm{GPa}$ [11], a nonunidirectional reinforcement correction factor, accounting only for fibers oriented in the direction of load

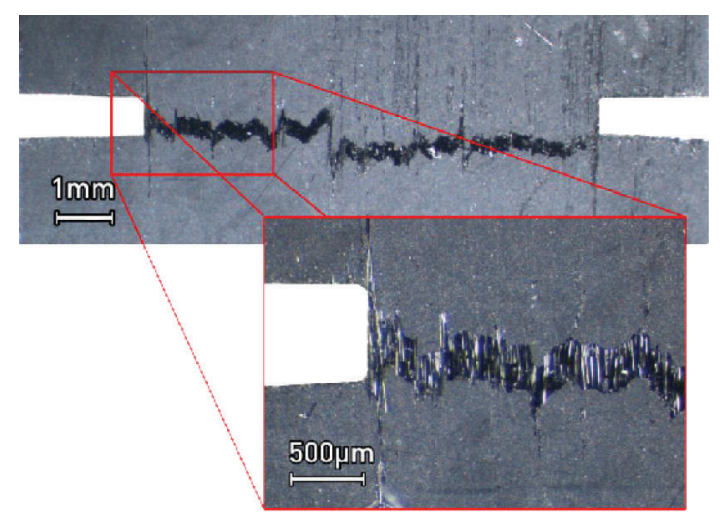

(a)

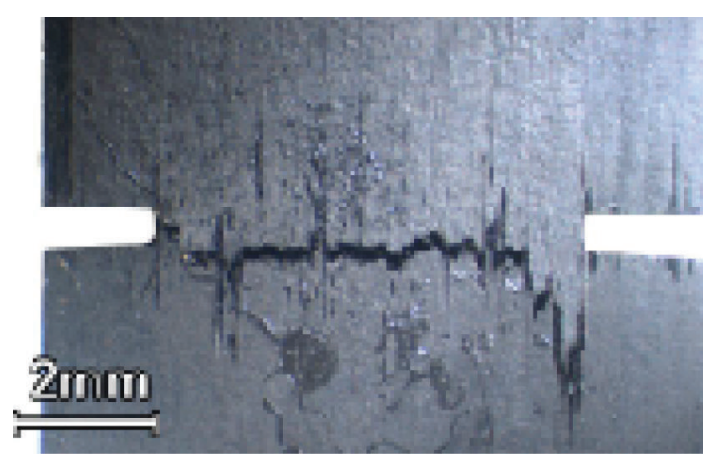

(b)

Figure 3: Postmortem optical stereoscope images of the notched lengths of a notched SiC/BMAS ceramic matrix composite demonstrating the dominant macrocrack and crack deflection path and magnification showing large-scale bridging and pullout phenomena.

application of $\lambda=0.5$ and a volume fraction of $V_{f}=$ 0.55 , is $106 \mathrm{GPa}$. As will be shown in the following sections, the observed difference between the true and theoretically expected moduli is associated with the thermal residual stress state of the composite. Composite strength also appeared to decrease with increasing notch length; average values of 270 , 280 , and $355 \mathrm{MPa}$ were calculated for specimens with notchto-width ratios of $0.35,0.2$, and 0 .

Substantial indications of large-scale bridging and pullout throughout the whole notched ligaments of tested specimens were observed postmortem, as the specimens were received in one free standing piece after removal from the grip with exposed fibers, which had apparently failed within the matrix environment and were pulling out, still bridging the two sides of the dominant macrocrack, as seen in Figure 3. The mere finding of a pullout mechanism active during composite testing indicates that the interfacial bond between the glass matrix and the $\mathrm{SiC}$ fibers was weak as anticipated by chemistry (see Section 2).

Loop hysteresis, a broadening unloading/reloading loop shape, was evident in all cycles apart from the initial couple that usually fell within the elastic behavior regime of the material. The effect, which also appeared coupled with the introduction of positive curvature on the unloading curves, 
is believed to be associated with frictional forces appearing during sliding of fibers across the matrix along the debonded length of the interface. At small strains, both composite constituents are able to sustain the applied because the coefficient of friction is still high-hence, no sliding occurs. As strain increases, fiber sliding begins. The maximum width of loops, a parameter that quantifies the magnitude of the hysteresis effect, increased with cycle count within the second-debonding regime and decreased again toward the final cycles in unnotched specimens, as seen in Figure 4. This is because during the last fracture stages, the cracking and debonding mechanisms have reached a saturated state, and the applied load is shared only by the intact fiber population.

The result of unloading/reloading cycles in the envelope curve to the mechanical behavior of the unnotched material is apparent in Figure 2 as an overall increase by $20 \%$ in attainable material stress. It is observed that the monotonic tension curve intersects the cyclic loading one exactly at the self-assembled CIP (0.3\% strain). Curve shape was similar for both cyclic and monotonically loaded specimens. At strains $<0.3 \%$, both testing conditions yielded comparable material response. At strains $>0.3 \%$, tangent modulus decreased more rapidly in the monotonic tension curve than in the cyclic tension curve. As a consequence, the debond region appeared at lower stresses in monotonic tension than in cyclic tension, still at the same strain of $0.3 \%$. The stiffening regime appeared at strains $>0.55 \%$ for both loading conditions. Within this last regime, the tangent modulus decreased more rapidly in monotonic than in cyclic tension curves. Average failure strain values of $0.85 \%$ were common for both loading conditions, a finding that suggest that the effect of cycling on the maximum deformation achievable by the composite is minimal. Based on the above results, it can be concluded that the mechanisms of matrix cracking, large-scale interfacial debonding, and ultimate stiffening are affected by the cyclic loading protocol.

3.2. Calculation of Residual Stress-Free Properties. It has previously been argued that the stress coordinate of CIPs assembled by extrapolation of the elastic compliance lines (upper linear part of reloading cycles) is equal to the average axial residual stress in the fibers multiplied by their volume fraction, whereas the strain coordinate corresponds to the axial residual strain in the fibres averaged over the gauge length [3]. Extending this argumentation to the experimentally self-assembled CIP of the current work, the axial residual stress and strain of $\mathrm{SiC}$ is calculated as $163 \mathrm{MPa}$ and $0.1 \%$, respectively.

The tensile nature of residual stresses suggests that longitudinal shrinkage of fibers was restricted by the surrounding matrix during cooling of the material from the firing temperature to ambient temperature. This can only hold if the glass matrix material has a CTE that is lower than the fibers. This is indeed the case, as CTEs for BMAS and SiC-Tyranno fibers are around $\alpha_{m}=2.5 \times 10^{-6} \mathrm{~K}^{-1}[12]$ and $\alpha_{f}=4.5 \times 10^{-6} \mathrm{~K}^{-1}$ (according to fiber manufacturer). For a composite with a noncracked matrix and perfect interfacial bonding, a classic theoretical estimation of TRS [5] is given as

$$
\sigma_{r}^{m}=E_{m} \frac{\lambda E_{f} V_{f}}{\lambda E_{f} V_{f}+E_{m} V_{m}}\left(a_{f}-a_{m}\right)\left(T_{o}-T_{p}\right),
$$

where $T_{o}=298 \mathrm{~K}$ and $T_{p}=1573 \mathrm{~K}$ are the operation and processing temperatures, respectively. Using the already known values of above parameters, the theoretical residual stress is found to be negative at $150 \mathrm{MPa}$ (compressive). Hence, the axial residual stress on the fibers would be tensile at $150 \mathrm{MPa}$. This value is only $8 \%$ less than the experimentally measured TRS.

If the CIP is to be considered as the TRS-free origin of the mechanical behavior of the composite, it would be interesting to examine the effect on properties of translating the whole curve in the stress-strain plane so that the CIP is brought into coincidence with the axis origin. This has the effect depicted in Figure 5 and results in a recalculation of the composite elastic at $104 \mathrm{GPa}$. The new value is $30 \%$ lower than the original modulus and much close to the average modulus of notched specimens, $113 \mathrm{MPa}$. The above example demonstrates the strong dependence of material properties on residual stress and suggests that quantification of residual stresses is key to reporting accurate and meaningful material properties.

It should not be overlooked that radial residual stresses also develop during material processing. These stresses influence the degree of load transfer between the fibers and the matrix across the interface; hence, they affect the amount of frictional energy dissipation during interfacial sliding, which is represented by the area of the hysteresis loops during cycling loading. However, this area does not affect the envelope tensile curve and the calculated elastic constants of the material.

3.3. Inelastic Strain. Composites tested in cyclic tension exhibited inelastic strain accumulation upon unloading, as per the example illustrated in Figure 2. This parameter is calculated as the strain offset at zero load just on the completion of unloading and is plotted in Figure 6 as a function of peak cycle stress, for specimen of different initial notch length. It is observed that notched specimens were associated with small values of positive inelastic strains which appeared to increase with notch length. A totally different effect is seen in unnotched specimens; there inelastic strain was negative and reached an absolute maximum value that was $400 \%$ higher than the inelastic strain of notched specimens. Negative strain accumulation originates from matrix crack closure occurring when the residual stress in the matrix is compressive (tensile in the fibers) and relief of residual stresses during loading causes an expansion of the matrix blocks and a contraction of fibers bridging the cracks. It was also found that inelastic strain increased with cycle count and attained a plateau value in the final cycles, where matrix cracking has completed. The latter observation suggests that inelastic strain is related to the matrix cracking mechanism, most possibly through the mechanical impediment of complete crack closure due to fiber roughness and/or contact between opposing faces 

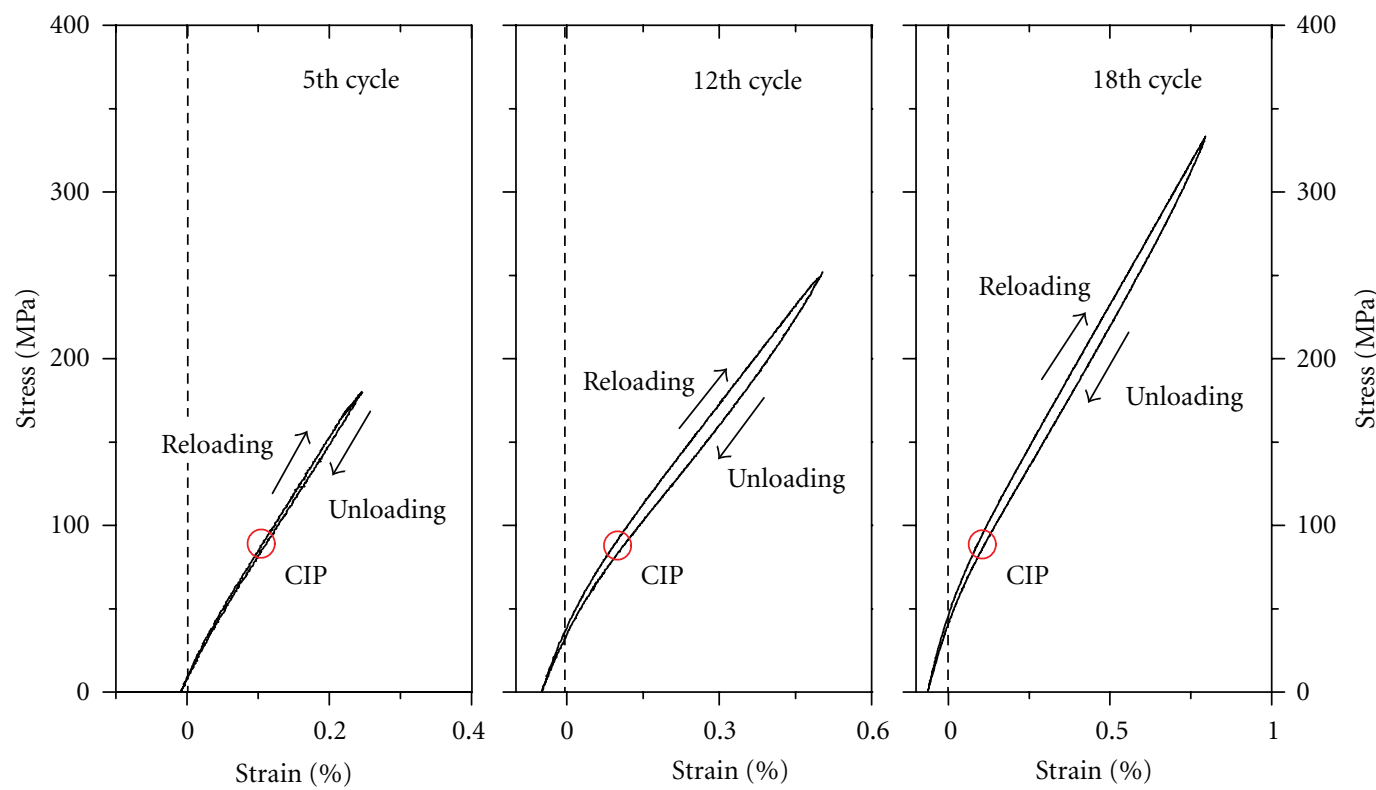

FIGURE 4: Evolution of hysteresis loop shape in the elastic, damage and stiffening regimes.

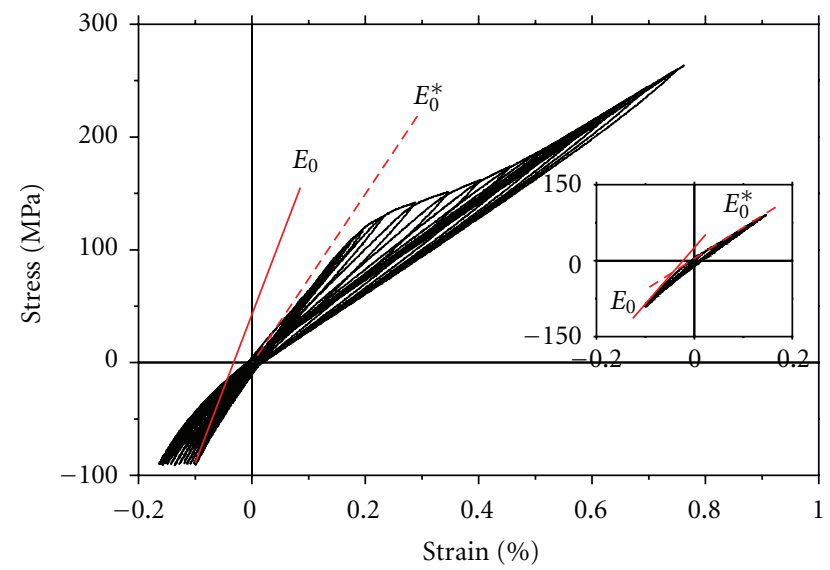

FIGURE 5: Translation of tensile curves for the calculation of TRSfree origin and associated properties such as elastic modulus.

microcrack slightly moved from their original positions during cyclic loading. This explanation is further favored by the increase in tangent modulus of the unloading curves towards the lower ends of the loops.

\section{Conclusions}

$\mathrm{SiC}_{f} / \mathrm{BMAS}$ ceramic matrix composites were tested under monotonic and cyclic tension with unloading/reloading cycles. The effects of the thermal residual stress state, presence of notches, and cycling were investigated. The mechanical

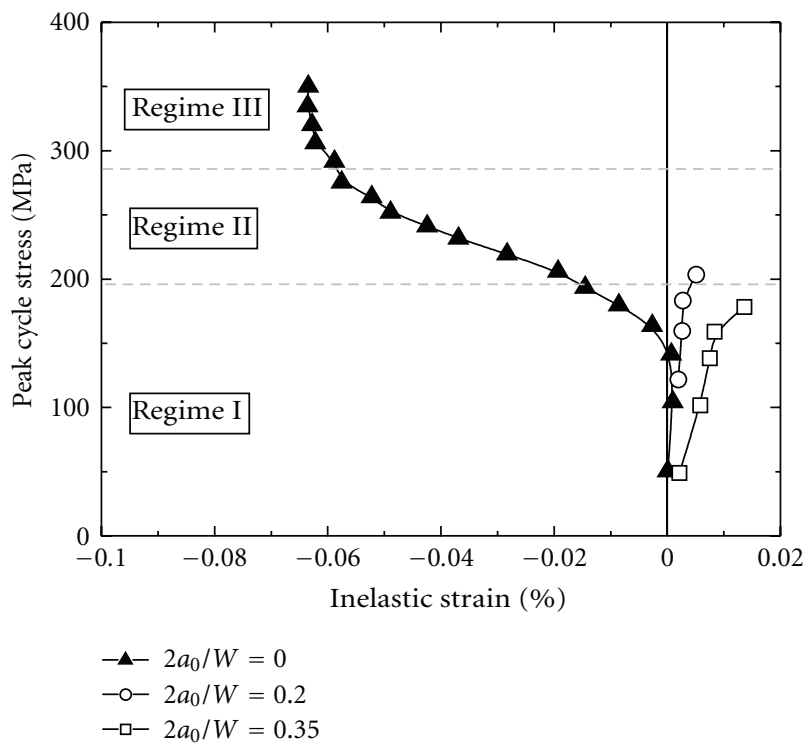

FIGURE 6: Inelastic strain plotted as a function of cycle stress for different notch length.

behavior of unnotched specimens was consistent with a "triple-regime" behaviour, wherein the elastic regime was followed by a softening effect attributed to large-scale interfacial debonding and a final prefracture, regime of apparent stiffening and increase in modulus due to load bearing by intact fibres in a completely cracked matrix. Notched specimens did not exhibit this latter regime. The thermal residual stress state of the composite was calculated as $163 \mathrm{MPa}$ from the coordinates of the common intersection 
point (CIP) of unloading-reloading cycles that appeared in the tensile curves of unnotched specimens. It was also found that the imposition of cyclic loading resulted in a material strengthening with respect to pure tension, with the envelope of the cyclic tension curves appearing at a maximum stress that was $20 \%$ higher than that achieved by specimens tested in monotonic tension. The effects of negative inelastic strain accumulation were also analyzed.

\section{References}

[1] R. F. Cooper and K. Chyung, "Structure and chemistry of fibre-matrix interfaces in silicon carbide fibre-reinforced glassceramic composites: an electron microscopy study," Journal of Materials Science, vol. 22, no. 9, pp. 3148-3160, 1987.

[2] P. M. Benson, K. E. Spear, and G. C. Pantano, "Interfacial characterisation of glass matrix/Nicalon $\mathrm{SiC}$ fiber composites: a thermodynamic approach," Ceramic Engineering and Science Proceedings, vol. 9, pp. 663-670, 1988.

[3] M. Steen, "Tensile mastercurve of ceramic matrix composites: significance and implications for modelling," Materials Science and Engineering A, vol. 250, no. 2, pp. 241-248, 1998.

[4] G. Camus, L. Guillaumat, and S. Baste, "Development of damage in a $2 \mathrm{D}$ woven $\mathrm{C} / \mathrm{SiC}$ composite under mechanical loading: I. Mechanical characterization," Composites Science and Technology, vol. 56, no. 12, pp. 1363-1372, 1996.

[5] H. Mei, "Measurement and calculation of thermal residual stress in fiber reinforced ceramic matrix composites," Composites Science and Technology, vol. 68, no. 15-16, pp. 3285-3292, 2008.

[6] M. Wang and C. Laird, "Characterization of microstructure and tensile behavior of a cross-woven C-SiC composite," Acta Materialia, vol. 44, no. 4, pp. 1371-1387, 1996.

[7] M. Wang and C. Laird, "Tension-tension fatigue of a crosswoven $\mathrm{C} / \mathrm{SiC}$ composite," Materials Science and Engineering A, vol. 230, no. 1-2, pp. 171-182, 1997.

[8] C. Cady, F. E. Heredia, and A. G. Evans, "In-plane mechanical properties of several ceramic-matrix composites," Journal of the American Ceramic Society, vol. 78, no. 8, pp. 2065-2078, 1995.

[9] G. N. Morscher, M. Singh, J. D. Kiser, M. Freedman, and R. Bhatt, "Modeling stress-dependent matrix cracking and stressstrain behavior in $2 \mathrm{D}$ woven $\mathrm{SiC}$ fiber reinforced CVI SiC composites," Composites Science and Technology, vol. 67, no. 6, pp. 1009-1017, 2007.

[10] D. Johnson-Walls, A. G. Evans, D. B. Marshall, and M. R. James, "Residual-stresses in machined ceramic surfaces," Journal of the American Ceramic Society, vol. 69, no. 1, pp. 44-47, 1986.

[11] S. Widjaja, "Determination of creep-induced residual stress in fiber-reinforced glass-ceramic matrix composites by X-ray diffraction," Materials Characterization, vol. 47, no. 1, pp. 47-54, 2001.

[12] U. S. Jais, W. E. Lee, and P. F. James, "Crystallization of barium osumilite glass," Journal of the American Ceramic Society, vol. 82, no. 11, pp. 3200-3208, 1999. 

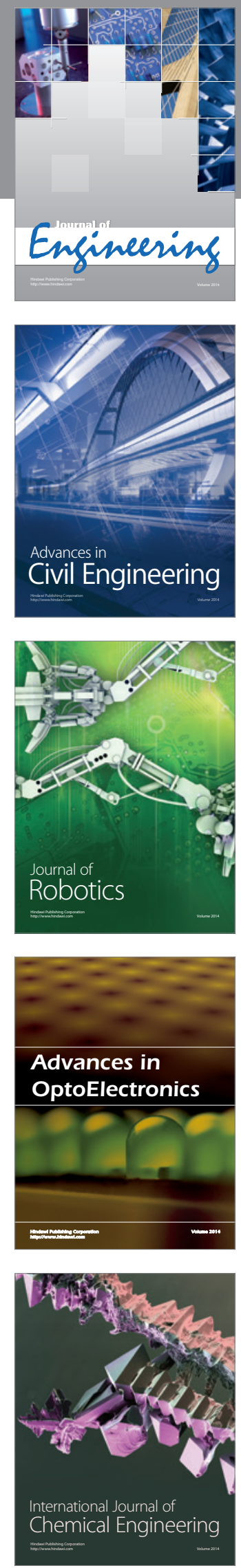

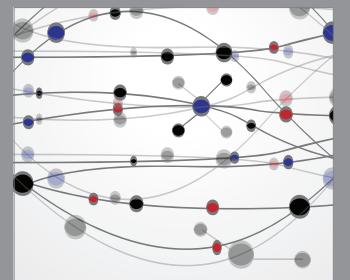

The Scientific World Journal
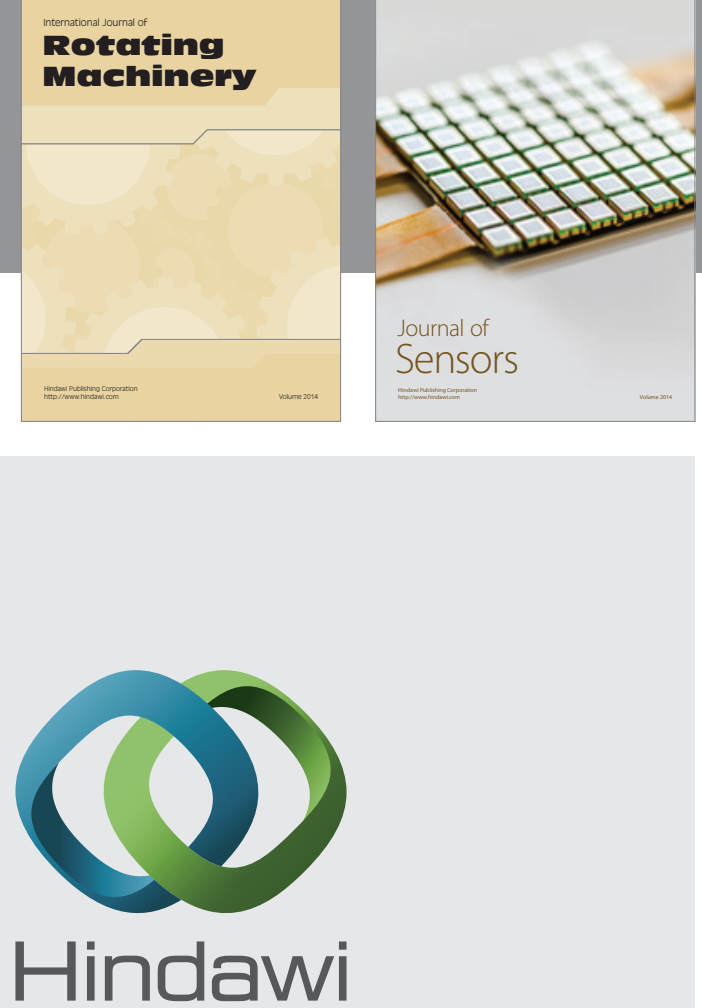

Submit your manuscripts at http://www.hindawi.com
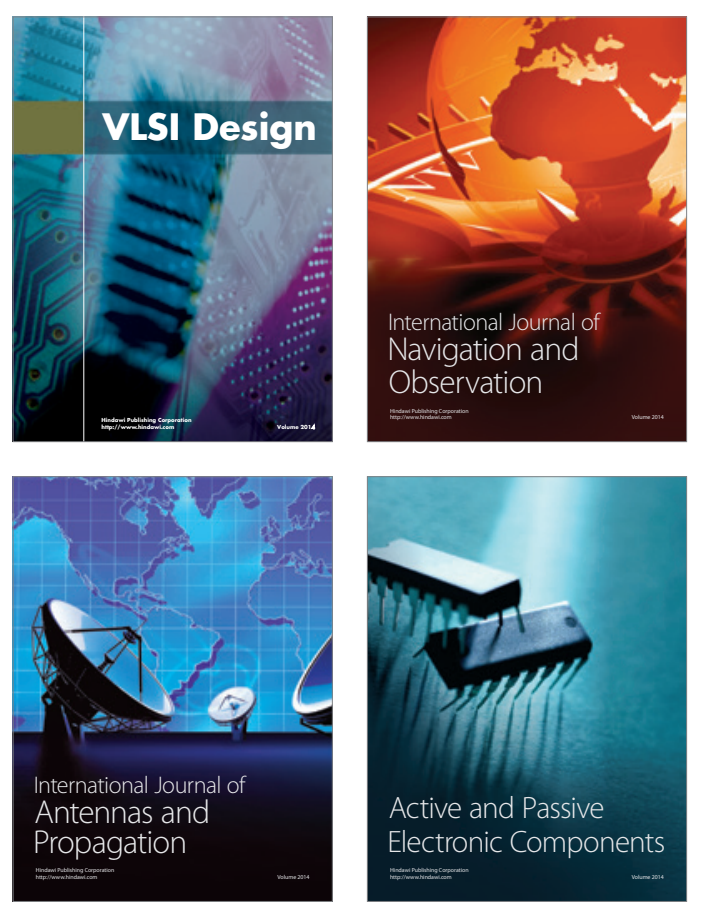
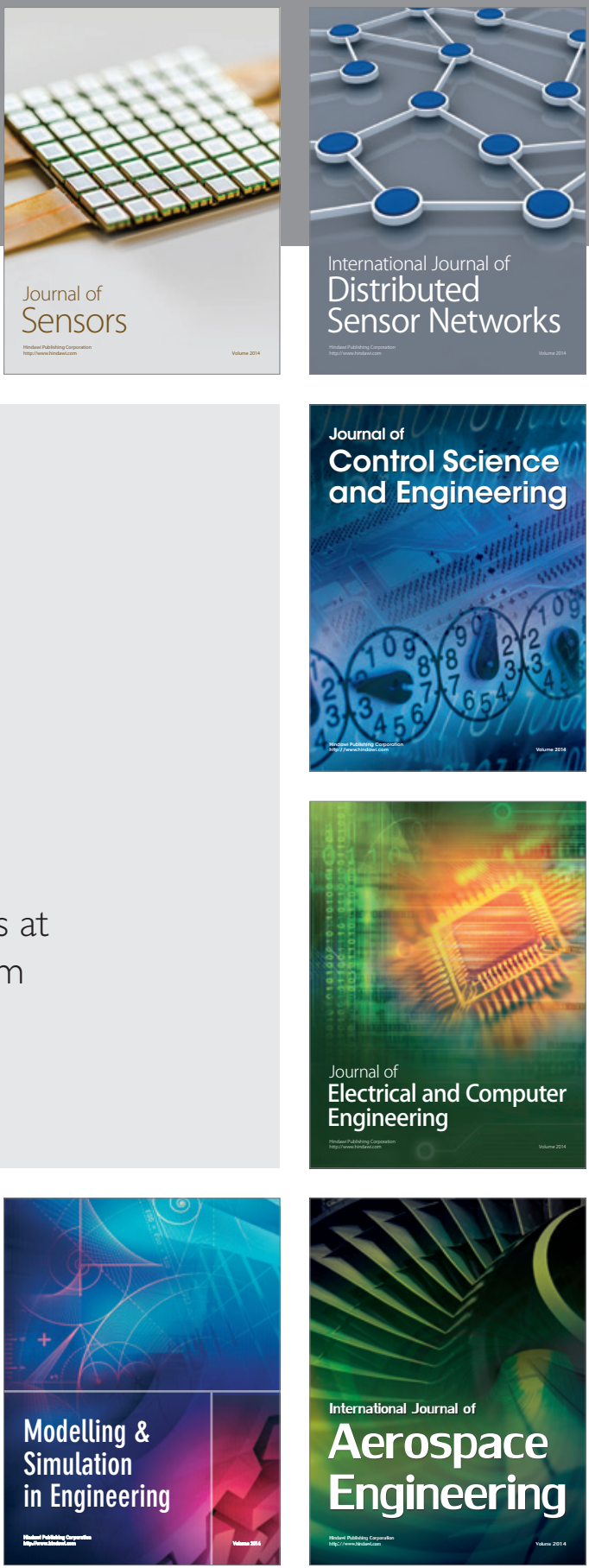

Journal of

Control Science

and Engineering
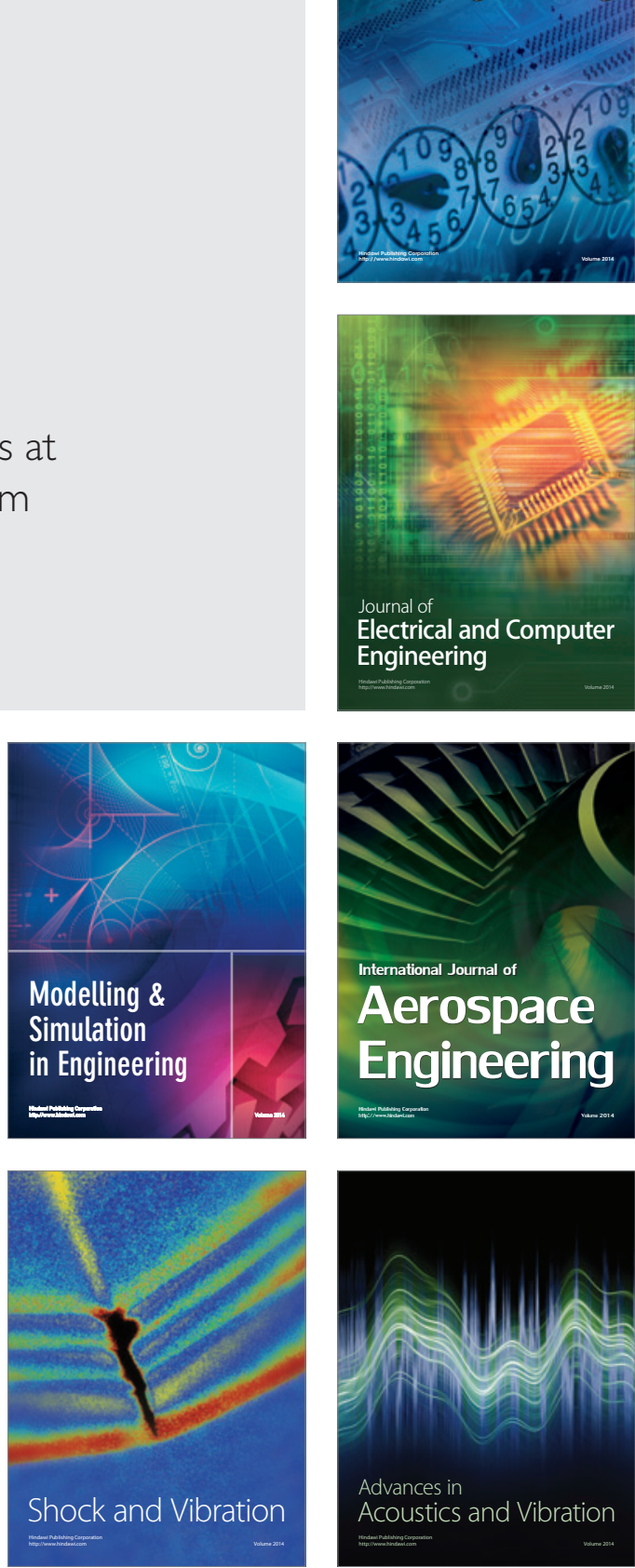